\title{
Pseudoscalar-Meson Form Factors: A Fresh Look by QCD Sum Rules
}

\author{
Irina Balakireva \\ D. V. Skobeltsyn Institute of Nuclear Physics, Moscow State University, 119991, Moscow, Russia \\ E-mail: iraxff@mail.ru
}

\section{Wolfgang Lucha*}

Institute for High Energy Physics, Austrian Academy of Sciences, Nikolsdorfergasse 18, A-1050

Vienna, Austria

E-mail: Wolfgang. Lucha@oeaw.ac.at

\section{Dmitri Melikhov}

Institute for High Energy Physics, Austrian Academy of Sciences, Nikolsdorfergasse 18, A-1050

Vienna, Austria,

Faculty of Physics, University of Vienna, Boltzmanngasse 5, A-1090 Vienna, Austria, and

D. V. Skobeltsyn Institute of Nuclear Physics, Moscow State University, 119991, Moscow, Russia

E-mail: dmitri_melikhov@gmx.de

Confronted with some surprising claims about the either experimentally measured or theoretically expected dependences on the involved momentum transfer of various form factors of pseudoscalar mesons, we reassess the present status of these objects by means of QCD sum rules. This approach provides well-developed and very efficient tools to relate in an analytical manner the parameters of quantum chromodynamics (QCD) - the quantum field theory that describes the strong interactions responsible for the formation of hadronic bound states - to the empirical features of such particles: Matrix elements of appropriately chosen products of interpolating currents that carry the quantum numbers of the hadrons under study are evaluated at both hadron and QCD level. In the latter case, all these nonlocal operators are expressed as series of local operators by Wilson's operator product expansion, with coefficients determined from perturbation theory. For vacuum expectation values, this introduces universal vacuum condensates that parameterize the nonperturbative contributions. Our ignorance about the higher hadron states is masked by quark-hadron duality assuming mutual cancellations of the contributions of hadronic excitations and continuum and of perturbative QCD beyond certain effective thresholds. Within this framework we show that a few theoretical findings for the charged-pion elastic form factor and one experimental result for the neutral-pion-to-photon transition form factor are at odds with very general, and likely sound, fundamental considerations.

Xth Quark Confinement and the Hadron Spectrum

8-12 October 2012

TUM Campus Garching, Munich, Germany

* Speaker. 


\section{Dispersive Local-Duality QCD Sum Rules for Pseudoscalar-Meson Form Factors}

We analyze the dependences on the involved momentum transfer squared $Q^{2}$ of the elastic form factor $F_{\pi}\left(Q^{2}\right)$ of the charged pion and of the form factor $F_{\pi \gamma}\left(Q^{2}\right)$ describing the transition $\pi^{0} \leftrightarrow \gamma \gamma^{*}$ of the neutral pion to a real and a virtual photon by dispersive QCD sum rules deduced from vacuum expectation values of products of the three adequate interpolating or electromagnetic currents $[1,2]$. In the limit of local duality (LD) [3] the sum rules relate the form factors to dispersion integrals over perturbatively deducible spectral densities. The $Q^{2}$-dependent upper integration limits, the effective thresholds $s_{\text {eff }}\left(Q^{2}\right)$, then encode the nonperturbative effects. In terms of strong coupling $\alpha_{\mathrm{s}}\left(Q^{2}\right)$ and $f_{\pi}=130 \mathrm{MeV}$, for $Q^{2} \rightarrow \infty$ factorization implies $Q^{2} F_{\pi}\left(Q^{2}\right) \rightarrow 8 \pi \alpha_{\mathrm{s}}\left(Q^{2}\right) f_{\pi}^{2}, Q^{2} F_{\pi \gamma}\left(Q^{2}\right) \rightarrow \sqrt{2} f_{\pi}$, and thus $s_{\text {eff }}(\infty)=4 \pi^{2} f_{\pi}^{2}$ [4]. Even if supported by quantum-mechanical solutions [5], modelling of $s_{\text {eff }}\left(Q^{2}<\infty\right)$ is non-trivial [6]. To sharpen our arguments, we define equivalent effective thresholds: sum rules with such integration limit reproduce experiment or any theoretical result exactly (Fig. 1).

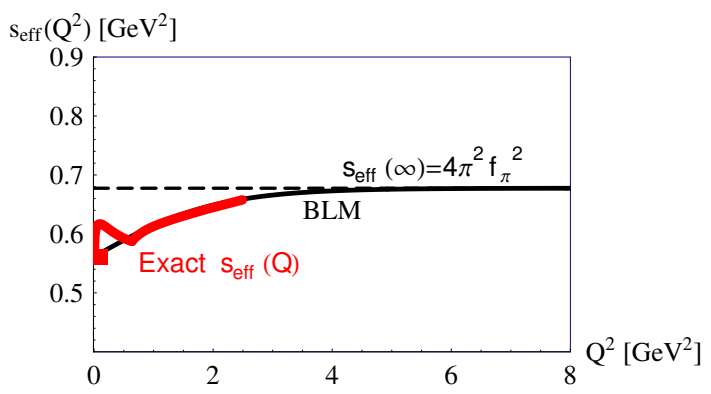

Figure 1: Naive LD modelling (BLM) [1] of the exact effective threshold for $F_{\pi}\left(Q^{2}\right)$ fixed by experiment [7].

\section{Charged-Pion Elastic and Neutral-Pion-to-Photon Transition Form Factors $[1,2]$}

Our model interpolates between the large- $Q^{2}$ asymptote and the empirical low- $Q^{2}$ behaviour of $s_{\text {eff }}\left(Q^{2}\right)$ [1] (Fig. 1). Not all other approaches [8] comply with the resulting form of $F_{\pi}\left(Q^{2}\right)$ (Fig. 2).
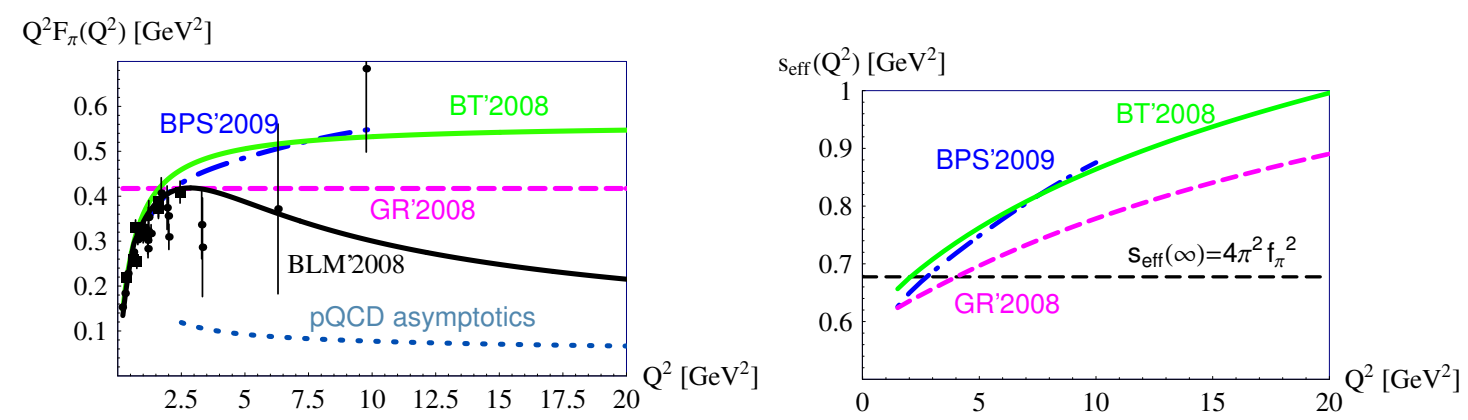

Figure 2: The $Q^{2}$ behaviour of the elastic pion form factor $F_{\pi}\left(Q^{2}\right)$ obtained [8] by Brodsky and de Téramond (BT'2008), Grigoryan and Radyushkin (GR'2008), and Bakulev, Pimikov, and Stefanis (BPS'2009)—and of the equivalent effective thresholds $s_{\text {eff }}\left(Q^{2}\right)$-is in clear conflict with LD model expectations [1] (BLM'2008). 
Regarding pseudoscalar-meson transition form factors, LD sum rules perform satisfactorily for $\left(\eta, \eta^{\prime}, \eta_{c}\right) \leftrightarrow \gamma \gamma^{*}$ but do not reproduce a BAB AR claim [9] of $Q^{2} F_{\pi \gamma}\left(Q^{2}\right)$ rising with $Q^{2}$ beyond the LD asymptote $\sqrt{2} f_{\pi}$ [1,2] (Fig. 3); confident in our approach, we feel that this mismatch casts some doubt on the BABAR measurement. Recent Belle observations [9] lend support to our point of view.
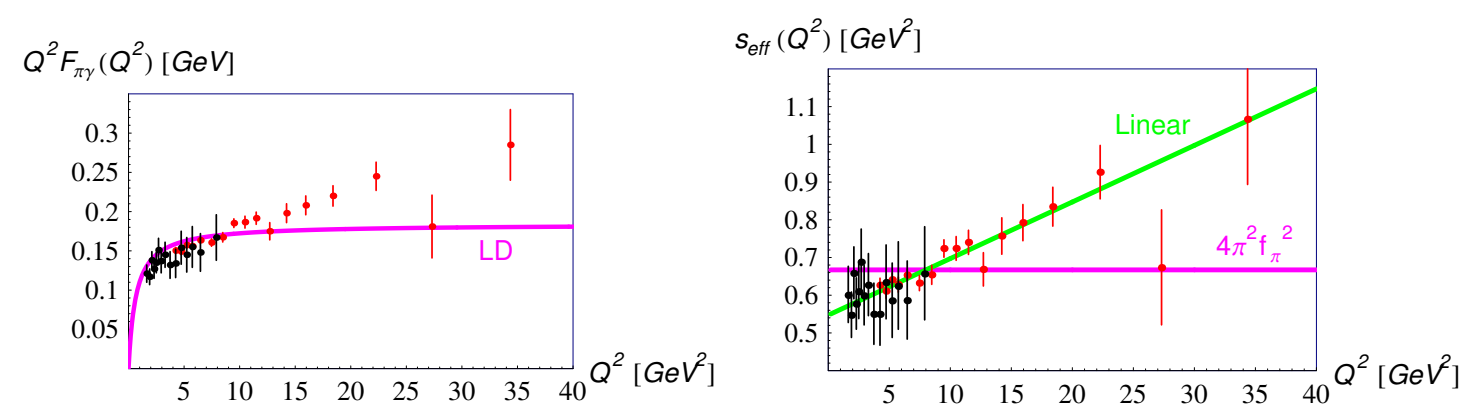

Figure 3: The BABAR results for $F_{\pi \gamma}\left(Q^{2}\right)$ [9] (red dots), unlike those by CELLO and CLEO (black dots) [9], are not compatible with LD sum-rule predictions as their incorporation would require a linear rise of $s_{\text {eff }}\left(Q^{2}\right)$.

Acknowledgments. D.M. was supported by the Austrian Science Fund (FWF), project no. P22843.

\section{References}

[1] V. Braguta, W. Lucha, and D. Melikhov, Phys. Lett. B 661 (2008) 354; I. Balakireva, W. Lucha, and D. Melikhov, J. Phys. G 39 (2012) 055007; Phys. Rev. D 85 (2012) 036006; Phys. Atom. Nucl. 76 (2013) (in press), arXiv:1203.2599 [hep-ph].

[2] W. Lucha and D. Melikhov, J. Phys. G 39 (2012) 045003; Phys. Rev. D 86 (2012) 016001; D. Melikhov and B. Stech, Phys. Rev. D 85 (2012) 051901(R); Phys. Lett. B (in press), arXiv:1206.5764 [hep-ph].

[3] V. A. Nesterenko and A. V. Radyushkin, Phys. Lett. B 115 (1982) 410; A. V. Radyushkin, Acta Phys. Pol. B 26 (1995) 2067.

[4] S. J. Brodsky and G. P. Lepage, Adv. Ser. Direct. High Energy Phys. 5 (1989) 93.

[5] W. Lucha and F. F. Schöberl, Int. J. Mod. Phys. C 10 (1999) 607.

[6] W. Lucha, D. Melikhov, and S. Simula, Phys. Rev. D 76 (2007) 036002; Phys. Lett. B 657 (2007) 148; Phys. Atom. Nucl. 71 (2008) 1461; Phys. Lett. B 671 (2009) 445; Phys. Rev. D 79 (2009) 096011; J. Phys. G 37 (2010) 035003; Phys. Lett. B 687 (2010) 48; Phys. Atom. Nucl. 73 (2010) 1770; J. Phys. G 38 (2011) 105002; Phys. Lett. B 701 (2011) 82; D. Melikhov, Phys. Lett. B 671 (2009) 450; W. Lucha, D. Melikhov, H. Sazdjian, and S. Simula, Phys. Rev. D 80 (2009) 114028.

[7] C. J. Bebek et al., Phys. Rev. D 17 (1978) 1693; T. Horn et al., Phys. Rev. Lett. 97 (2006) 192001; V. Tadevosyan et al., Phys. Rev. C 75 (2007) 055205; G. M. Huber et al., Phys. Rev. C 78 (2008) 045203.

[8] S. J. Brodsky and G. F. de Téramond, Phys. Rev. D 77 (2008) 056007; H. R. Grigoryan and A. V. Radyushkin, Phys. Rev. D 78 (2008) 115008; A. P. Bakulev, A. V. Pimikov, and N. G. Stefanis, Phys. Rev. D 79 (2009) 093010.

[9] H. J. Behrend et al., Z. Phys. C 49 (1991) 401; J. Gronberg et al., Phys. Rev. D 57 (1998) 33; B. Aubert et al., Phys. Rev. D 80 (2009) 052002; S. Uehara et al., Phys. Rev. D 86 (2012) (in press), arXiv:1205.3249 [hep-ex]. 